\title{
Lactocollybia subvariicystis, a new species of little known genus Lactocollybia from subtropical south China
}

\author{
Hosen $\mathrm{MI}^{1}, \mathrm{Li} \mathrm{TH}^{1}$, Chen $\mathrm{XN}^{1,2}$ and Deng $\mathrm{WQ}^{1}$ \\ ${ }^{\text {I}}$ State Key Laboratory of Applied Microbiology Southern China, Guangdong Provincial Key Laboratory of Microbial \\ Culture Collection and Application, Guangdong Institute of Microbiology, Guangzhou 510070, CHINA. \\ mycolab@263.net,iqbalmyco@gmail.com \\ ${ }^{2}$ South China Agricultural University, Guangzhou 510642, China
}

Hosen MI, Li TH, Chen XN, Deng WQ. 2016 - Lactocollybia subvariicystis, a new species of little known genus Lactocollybia from subtropical south China. Mycosphere 7(6), 794-800, Doi 10.5943/mycosphere/7/6/10

\begin{abstract}
Lactocollybia is poorly known in China. Prior to this report, only a single species of this genus was reported from tropical Yunnan Province in the country. In this study, Lactocollybia subvariicystis is reported as a new species. It is characterized by its mycenoid habit with white basidiomata, white, adnate to sinuate lamellae, numerous gloeocystidia in pileus-stipe context, commonly present clamp connections, and association with living trunks of Acacia confusa. Although the phylogenetic relationships of Lactocollybia with other genera remain unclear to science, the taxonomic position of the new species within the genus is clear with morphological and molecular evidence. It is a member of genus Lactocollybia sect. Albae. Description, color photographs, comparison with phenotypically similar taxa and line drawings are presented.
\end{abstract}

Key words - Marasmiaceae - molecular phylogeny - subtropical fungus - taxonomy

\section{Introduction}

Lactocollybia Singer is a little known genus in the family Marasmiaceae. The genus has five sections: i. sect. Bertrandiella (Heim) Singer, ii. sect. Lactocollybia Singer, iii. sect. Albae Singer, iv. sect. Aurantiacae Singer and v. sect. Graminicolae Singer. These are classified based on their various characters such as habit of the carpophores (collyboid, pleurotoid, mycenoid and omphalioid), presence or absence of latex, subhymenium structure, clamp connections, gloeocystidia, pigmentation of the basidiomata, and living (on living cortex of trees, on cycads, fallen fruits, etc.) or lignicolous habitat (Singer 1986).

Nineteen Lactocollybia species have been reported worldwide up to date (www.indexfungorum.com, Nov. 2016). They are mainly distributed in tropical to subtropical regions, and few of the species can be found in the warm temperate zones in North America and South America (Singer 1986). In China, only a single species L. epia (Berk. \& Br.) Pegler has been reported from tropical area of Yunnan Province before (Yang 2000). In the present study, another species of the genus was found to be a new species to science, which is mycenoid in habit and found on living trunks of Acacia confusa Merr. 
Molecular study for the genus Lactocollybia had not yet been studied. Only few sequences of the nuclear ribosomal internal transcribed spacer (ITS) including partial sequences of nuclear ribosomal large subunit (nrLSU) are available for this genus. In this study, three nuclear ribosomal DNA sequences were generated from the newly collected specimens of Lactocollybia. The species is delimitated with morphological data and molecular evidence of ITS sequence analysis.

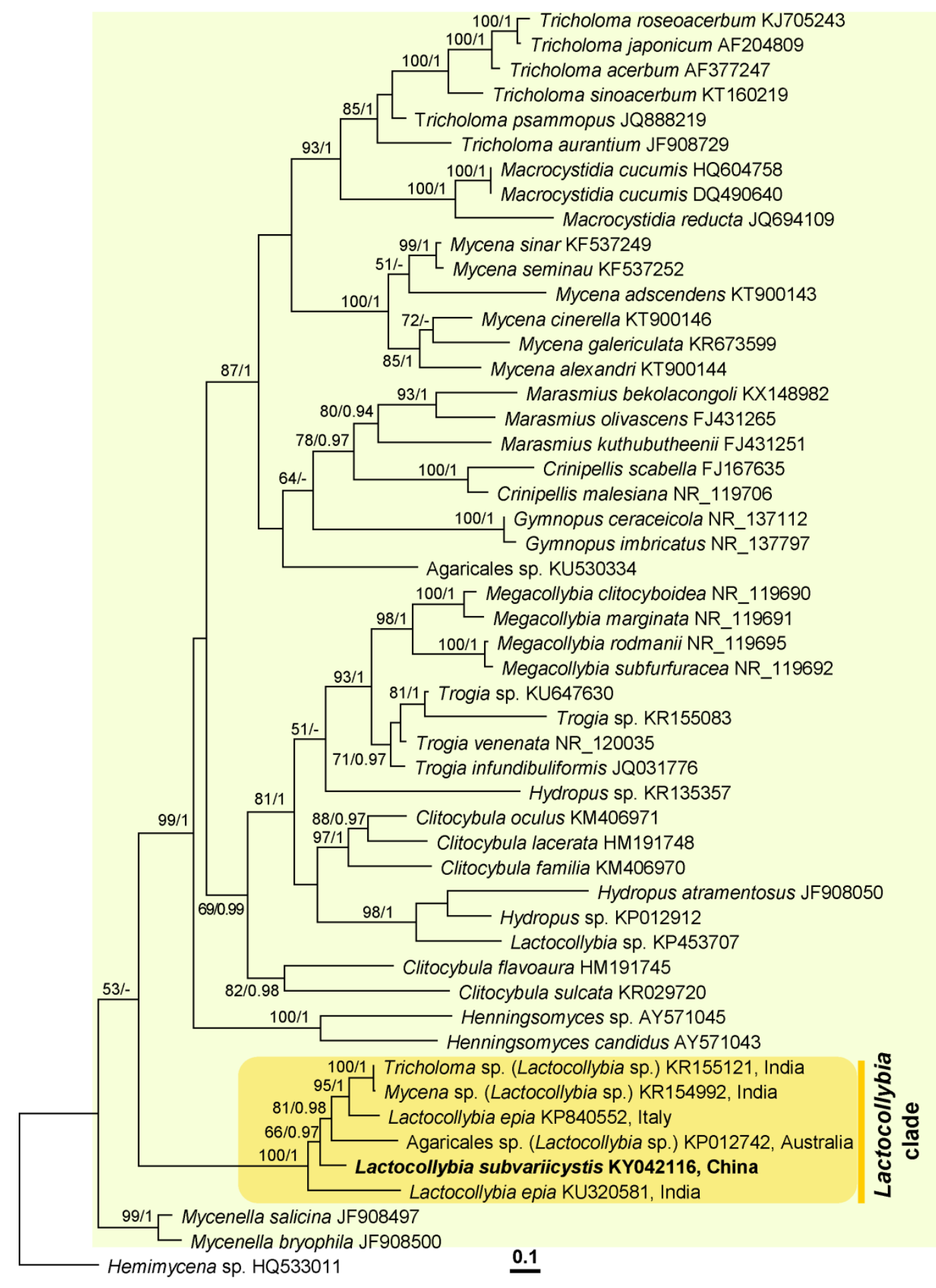

Fig. 1 - RAxML tree and phylogenetic relationships of Lactocollybia inferred from ITS sequences analysis. RAxML bootstrap supports values $(>50 \%)$ and Bayesian posterior probabilities $(>0.90)$ are indicated on branches. Hemimycena sp. is rooted as outgroup. Newly generated sequence for $L$. subvariicystis is highlighted in bold. GenBank accession numbers are provided after the species name. 


\section{Materials \& methods}

\section{Morphological methods}

Macro-morphological descriptions were based on the field notes and photos of basidiomata. Color codes mentioned in the description are from Kornerup \& Wanscher (1978). The examined materials were collected along the road sides close to Huanghuagang Commemoration Park, Guangzhou, China, and deposited in the Fungal Herbarium of Guangdong Institute of Microbiology (GDGM), Guangzhou, China. Micro-morphological observations were done from the dried specimens and methodology followed those in Hosen et al. (2016a, b).

\section{Molecular methods}

DNA extraction and PCR protocols are as those used in Hosen et al. (2013). ITS1/ ITS4 (White et al. 1990) and LROR/LR5 (Vilgalys \& Hester 1990) primer pairs were used to amplify the nuclear ribosomal internal transcribed (ITS) region. PCR products were purified with PCR Purification Kit (Sangon Biotech Co., Shanghai). Purified PCR products were sent to commercial sequencing company for sequencing (Majorbio, Guangzhou, China). Sequences were blasted against NCBI nucleotide database and closest sequences were downloaded together with other taxa of Hydropoid clade (Clitocybula (Singer) Singer ex Métrod, Henningsomyces Kuntze, Hydropus Kühner ex Singer and Megacollybia Kotl. \& Pouzar and) of Matheny et al. (2006). Additionally, some representative taxa of Crinipellis Pat., Gymnopus (Pers.) Gray, Hemimycena Singer, Macrocystidia Joss., Marasmius Fr., Mycenella (J.E. Lange) Singer, Tricholoma (Fr.) Staude and Trogia Fr., were retrieved from GenBank in order to see the close relativeness of Lactocollybia in molecular phylogenetic analysis. Hemimycena was selected as outgroup for molecular phylogenetic analysis. The matrix was aligned with MAFFT (Katoh et al. 2005). Maximum likelihood phylogenetic tree was generated using RAxML v.7.2.6 (Stamatakis 2006) with 100 bootstrap replications, and supported with posterior probabilities using MrBayes v.3.1.2 (Ronquist \& Huelsenbeck 2003).

\section{Results}

\section{Molecular phylogenetic results}

In this study, three sequences (ITS: KY042116, nrLSU: KY042117 and KY042118) were generated from the newly collected materials of Lactocollybia and deposited in the GenBank. Only the ITS sequence was used for the reconstruction of a molecular phylogenetic tree, and nrLSU sequences were deposited to GenBank for future reference. Percent identity between $L$. subvariicystis and L. epia is $87-88$ based on blast search results of ITS sequence; while nrLSU sequence from the Chinese collection L. subvariicystis is $97 \%$ identical to those of Italian L. epia (KP840552). The ITS dataset includes 51 fungal sequences and consisting of 981 nucleotide sites (gaps included) of which 604 are parsimony informative characters. The aligned ITS dataset is deposited in TreeBASE (S119966). ITS sequence of the Chinese L. subvariicystis nests in a clade with those of L. epia and supported by both ML and BI analyses (100\% ML BS, 1.0 PP). The summarized result of this analysis is presented in Fig. 1.

\section{Taxonomy}

Lactocollybia subvariicystis Iqbal Hosen \& T.H. Li, sp. nov. Figs $2-3$ MycoBank MB 819173

Diagnosis - Differs from L. variicystis in having obviously longer but narrower basidiospores (8-) 8.5-9.5(-10.5) $\times 4.5-5.3 \mu \mathrm{m}$, not or hardly plicate margin and habitat with A. confusa.

Etymology - The epithet "subvariicystis" (Lat.) refers to the morphology that is similar to $L$. variicystis. 

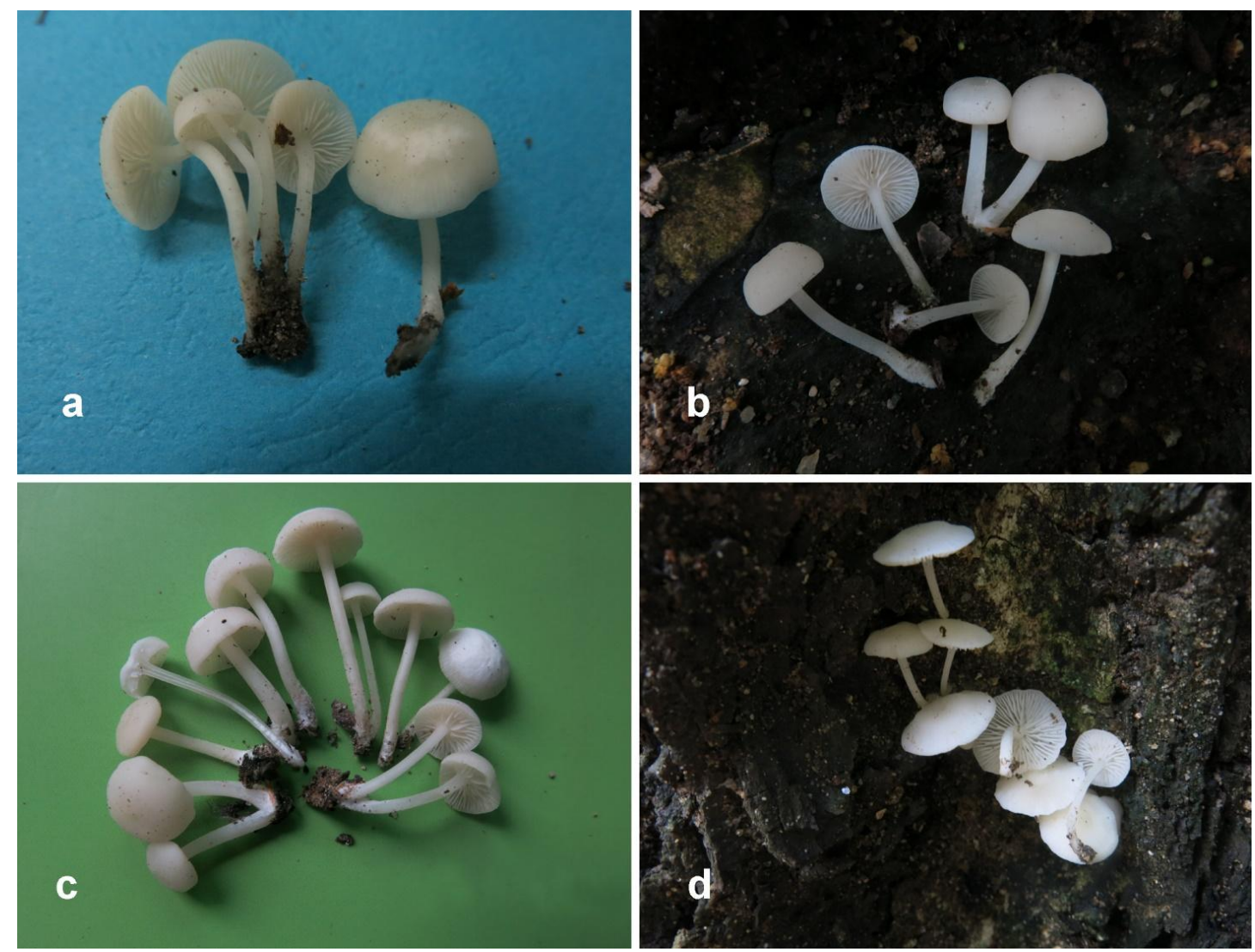

Fig. 2 - Basidiomata of Lactocollybia subvariicystis. a. Iqbal 911 (GDGM 46535). b-c. Iqbal 912 (GDGM 46536, holotype). d. Iqbal 913 (GDGM 46537, paratype).

Typification - CHINA. Guangdong Province, Guangzhou, Huanghuagang Commemoration Park, 10 Sep 2016, Iqbal 912 (GDGM 46536, holotype).

Basidiomata small-sized, mycenoid. Pileus 10-20 mm broad, hemispherical then expanding, becoming convex or flattened with age, smooth, dry, hygrophanous, slightly depressed at disc in some pilei, milk white (1A1), white to creamy-white (1A1), without striation or hardly plicate at the margin in some pilei; context $1.2 \mathrm{~mm}$ thick at the center of pileus, white, unchanged when cut or injured. Lamellae $12-17 \times 1.5-2 \mathrm{~mm}$, adnexed to sinuate, subdistant, up to $1 \mathrm{~mm}$ wide, not or occasionally forked or not anastomosing, white to creamy white (1A1); lamellulae common, with several ranks and various widths. Stipe $20-30 \times 1-1.5 \mathrm{~mm}$, cylindrical, slightly thickening towards base, central to slightly eccentric, decurved, smooth but very fine pruinose under a lens, white to creamy-white (1A1), fistulose, unchanged when cut or injured; basal mycelium white.

Basidiospores [60/3/3] (8-)8.5-9.5(-10.5) × 4.5-5.3 $\mu \mathrm{m},\left[\mathrm{Q}=1.65-1.81(-1.92), \mathrm{Q}_{\mathrm{m}}=1.74\right.$ $\pm 0.10]$, amygdaliform to broadly fusoid, thin-walled, pale yellow when observed in $\mathrm{H}_{2} \mathrm{O}$ and $5 \%$ $\mathrm{KOH}$, inamyloid, smooth, apiculus conspicuous up to $0.7 \mu \mathrm{m}$ long. Basidia 22-27 $\times 6-7 \mu \mathrm{m}$, clavate, hyaline and pale yellow in $\mathrm{H}_{2} \mathrm{O}$, thin-walled, mostly 4-spored, occasionally 2- or 3-spored, with sterigmata up to $5 \mu \mathrm{m}$ long; subhymenium thin, with subcellular to ramose hyphae. Hymenophoral trama regular, densely arranged parallel hyphae 4-10 $\mu \mathrm{m}$ wide. Pileal tramma interwoven, composed of 5-12 $\mu \mathrm{m}$ wide hyphae, mixed with abundant fusoid gloeocystidia which are sharply attenuated towards ends. Cheilo- and pleurocystidia 30-50 $\times 9-13 \mu \mathrm{m}$, crowded, variable in shape, cylindrical to subcylindrical, fusoid to subfusoid with mucronate apex, lageniform with long necked up to $30 \mu \mathrm{m}$. Pileipellis a cutis, composed of hyaline hyphae and refractive gloeocystidia; hyphae 3-8 $\mu \mathrm{m}$ wide, hyaline, smooth, thin-walled; gloeocystidia numerous, fusoid 10-18 $\mu \mathrm{m}$ wide, abruptly tapering at both ends, often forked, refractive, with vacuolar pigmentation. Stipe trama composed of parallel hyphae 6-12 $\mu \mathrm{m}$ wide, colorless or pale yellow, mixed with abundant fusoid gloeocystidia. Caulocystidia present similar to cheilocystidia but smaller. Clamp connections common. 


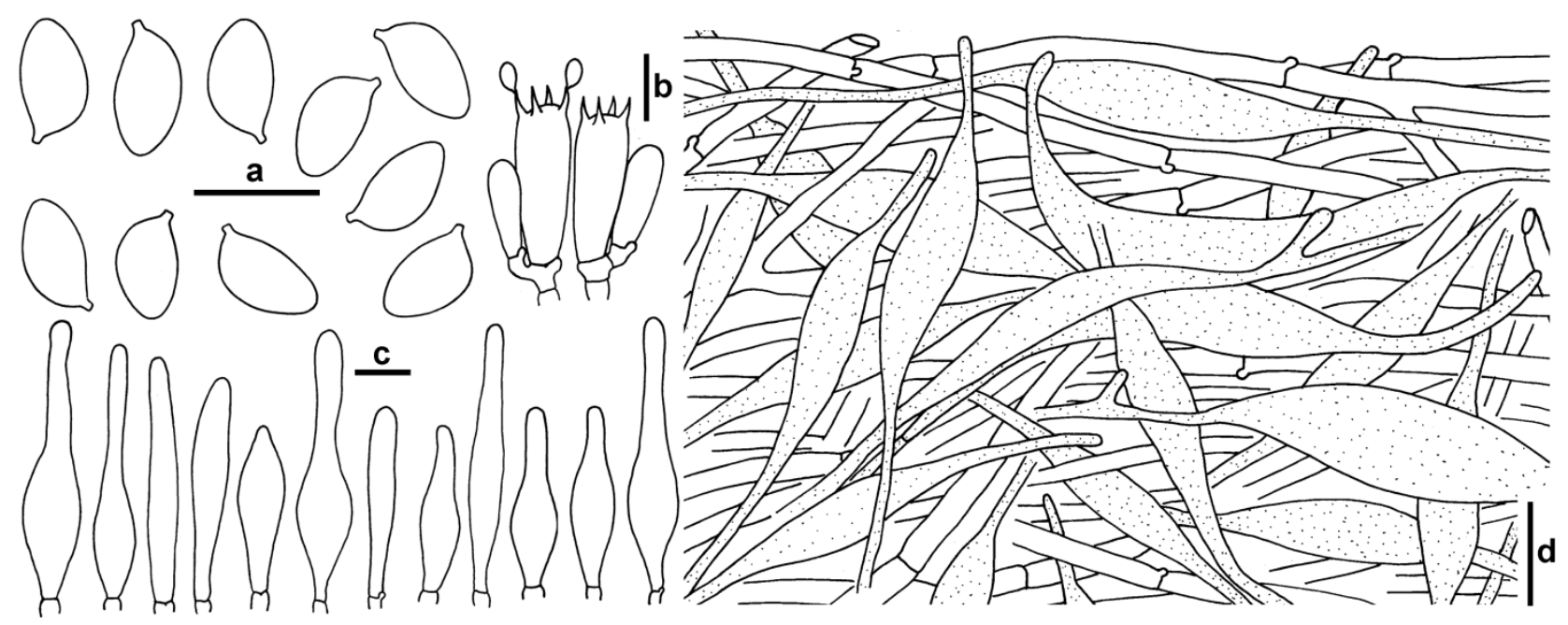

Fig. 3 - Microscopic features of Lactocollybia subvariicystis (Iqbal 912, GDGM 46536, holotype). a. Basidiospores. b. Basidia with basidioles. c. Cheilo- and pleurocystidia. d. Pileipellis. Scale bars: $\mathrm{a}-\mathrm{c} 10 \mu \mathrm{m}, \mathrm{d} 20 \mu \mathrm{m}$.

Known distribution - Known only from the type locality in China.

Habit and habitat - Solitary, caespitose or in small groups on the standing living trunks of $A$. confusa.

Materials examined - China. Guangdong Province, Guangzhou, along the roadsides around a bus stop station at Huanghuagang Commemoration Park, 9 Sep 2016, Iqbal 911 (GDGM 46535); same location; same location, 12 Sep 2016, Iqbal 913 (GDGM 46537, paratype).

\section{Discussion}

Lactocollybia subvariicystis is characterized by its small, white, hygrophanous basidiomata, sinuate to adnexed lamellae, common presence of fusoid gloeocystidia in the context of pileus, lamellae and stipe which is sharply tapering at both ends, and associated with the living tree of $A$. confusa. This species is a member of sect. Albae because of having subcellular to ramose elements in subhymenium, abundant clamps connection, numerous gloeocystidia, and habitat on living trees (Singer 1986).

The phylogenetic relationships of Lactocolybia remained unknown despite modern species delimitation are based on molecular approaches. Most reports for Lactocollybia were from morphological based studies. For instance, only sequences for L. epia are available in GenBank out of 19 species of this genus. In the phylogenetic analysis, the Chinese L. subvariicystis grouped with L. epia from Italy and India, Tricholoma sp. (KR155221)/Mycena sp. (KR154992) from India, and one Agaricales sp. (KP012742) from Australia with maximum BS support (Fig. 1). It should be mentioned that other Tricholoma or Mycena species are nested in other clades, quite far from Lactocollybia clade with strong BS supports (Fig. 1). Furthermore, Tricholoma has no clamp connections and cystidia (Singer 1986), while Mycena has amyloid basidiospores (Singer 1986) which are not fitted with the taxonomic concept of Lactocollybia. The substantial taxonomic treatments for the Indian and Australian collections are not available to compare with Lactocollybia. Based on the present molecular results, it may conclude that the uploaded ITS sequences to GenBank for Mycena sp. (KR154992), Tricholoma sp. (KR155221) and Agaricales sp. (KP012742) might be the members of Lactocollybia. Previously Lactocollybia was supposed to be a close relative to Macrocystidia (Singer 1986) because of the numerous gloeocystidia, abundant cystidia and inamyloid basidiospores (Singer 1986). However, in the present study, they have not significantly close relativeness to each other, being nested in two different clades (Fig. 1). Interestingly, one collection from Mexico (TENN 055867) is deposited in GenBank (KP453707) as Lactocollybia sp. is grouped with Hydropus species with strong BS support (98\% ML BS, 1.0 PP; Fig. 1), which may not be a true Lactocollybia (Marisol Sanchez-Garcia, pers. comm.). Besides the 
sequence of 'L. epia KP840552' from Italy and that of 'L. epia KU320581' from India should represent two different species of Lactocollybia, since the sequences have only $83 \%$ identity. The phylogenetic relationships of Lactocollybia spp. within the genus or other genera remain unresolved because only limited sequences of Lactocollybia are available until now.

Lactocollybia variicystis, originally reported from South Africa (Reid \& Eicker 1998), is morphologically close to the new species $L$. subvariicystis from subtropical south China. However, the South African species differs from the Chinese one in having obviously shorter but broader basidiospores measuring 6.6-8 $\times 4-6 \mu \mathrm{m}$, and found on the stump of Salix sp. Lactocollybia pilicystis D.A. Reid \& Eicker, also originally reported from South Africa, although has small-sized white basidiomata and commonly present of gloeocystidia like L. subvariicystis, differs from the latter in having a usually longer stipe up to $120 \mathrm{~mm}$, rare or scanty of pleurocystidia, and much smaller basidiospores measuring 4.75-6.2(-6.75) × 3.2-4.5 $\mu \mathrm{m}$ (Reid \& Eicker 1998).

Previously, only a single species of the genus, i.e. L. epia has been reported from Yunnan Province in China (Yang 2000). The Chinese collection L. epia also has similar color of basidiomata but differs considerably from the new species $L$. subvariicystis in having closer adnate to decurrent lamellae, longer and thicker stipe $(10-60 \times 2-5 \mathrm{~mm})$, fusiform to occasionally clavate or subcylindrical cystidia, shorter but broader basidiospores 6.5-8.5 × 4-5.5(-6) $\mu \mathrm{m}$ (Yang 2000). Furthermore, Yang (2000) suggested that the Chinese 'L. epia' collection is more closely related to L. cycadicola (Joss.) Singer, if they are not conspecific.

\section{Acknowledgements}

The authors are thankful to Drs. Pierre-Arthur Moreau (France), Marisol Sanchez-Garcia (USA) and Alfredo Vizzini (Italy) for their online discussion on the genus Lactocollyia. This work was financed by the National Natural Science Foundation of China (31260011 and 31570020).

\section{References}

Hosen MI, Feng B, Wu G, Zhu, XT, Li YC, Yang ZL. 2013 - Borofutus, a new genus of Boletaceae from tropical Asia: morphology, taxonomy and phylogeny. Fungal Diversity 58, 215-226.

Hosen MI, Li TH, Li T, Zhong XJ, Chen Y. 2016a - Tricholoma sinoacerbum, a bitter species from Guangdong Province of China. Mycoscience 57, 233-238.

Hosen MI, Li TH, Lodge DJ, Rockefeller A. 2016b - The first ITS phylogeny of the genus Cantharocybe (Agaricales, Hygrophoraceae) with a new record of C. virosa from Bangladesh. MycoKeys 14, 37-50.

Katoh K, Kuma K, Toh H, Miyata T. 2005 - MAFFT version 5: improvement in accuracy of multiple sequence alignment. Nucleic Acids Research 33, 511-518.

Kornerup A, Wanscher JH. 1978 - Methuen handbook of colour. 3rd edn. Eyre Methuen, London.

Matheny PB, Curtis JM, Hofstetter V, Aime MC, Moncalvo JM, Ge ZW, Slot JC, Ammirati JF, Baroni TJ, Bougher NL, Hughes KW, Lodge DJ, Kerrigan RW, Seidl MT, Aanen DK, DeNitis M, Daniele GM, Desjardin DE, Kropp BR, Norvell LL, Parker A, Vellinga EC, Vilgalys R, Hibbett DS. 2006 - Major clades of Agaricales: a multilocus phylogenetic overview. Mycologia 98, 982-995.

Reid DA, Eicker A. 1998 - South African fungi 6. The genus Lactocollybia (Basidiomycota) in South Africa. Mycotaxon 66, 153-163.

Ronquist F, Huelsenbeck JP. 2003 - MrBayes 3: Bayesian phylogenetic inference under mixed models. Bioinformatics 19, 1572-1574.

Singer R. 1986 - The Agaricales in Modern taxonomy. 4th ed. Koeltz Scientific Books. Koenigstein.

Stamatakis A. 2006 - RAxML-VI-HPC: maximum likelihood-based phylogenetic analyses with thousands of taxa and mixed models. Bioinformatics 22, 2688-2690. 
Vilgalys R, Hester M. 1990 - Rapid genetic identification and mapping of enzymatically amplified ribosomal DNA from several Cryptococcus species. Journal of Bacteriology 172, 4238-4246.

White TJ, Bruns T, Lee S, Taylor J. 1990 - Amplification and direct sequencing of fungal ribosomal RNA genes for phylogenetics. In: Innis MA, Gelfand DH, Sninsky JJ, White TJ, eds. PCR protocols: a guide to methods and applications. California: Academic Press. p 315322.

Yang ZL. 2000 - Notes on five common but little known higher Basidiomycetes from tropical Yunnan, China. Mycotaxon 74, 45-56. 